\title{
High-performance InGaZnO-based ReRAMs
}

DOI:

10.1109/TED.2019.2912483

\section{Document Version}

Accepted author manuscript

Link to publication record in Manchester Research Explorer

\section{Citation for published version (APA):}

Ma, P., Liang, G., Wang, Y., Li, Y., Xin, Q., Liu, Y., \& Song, A. (2019). High-performance InGaZnO-based ReRAMs. IEEE Transactions on Electron Devices, 66(6), 2600. https://doi.org/10.1109/TED.2019.2912483

\section{Published in:}

IEEE Transactions on Electron Devices

\section{Citing this paper}

Please note that where the full-text provided on Manchester Research Explorer is the Author Accepted Manuscript or Proof version this may differ from the final Published version. If citing, it is advised that you check and use the publisher's definitive version.

\section{General rights}

Copyright and moral rights for the publications made accessible in the Research Explorer are retained by the authors and/or other copyright owners and it is a condition of accessing publications that users recognise and abide by the legal requirements associated with these rights.

\section{Takedown policy}

If you believe that this document breaches copyright please refer to the University of Manchester's Takedown Procedures [http://man.ac.uk/04Y6Bo] or contact uml.scholarlycommunications@manchester.ac.uk providing relevant details, so we can investigate your claim.

\section{open 2 Access}




\title{
High-performance InGaZnO-based ReRAMs
}

\author{
Pengfei Ma, Guangda Liang, Yiming Wang, Yunpeng Li, Qian Xin, Yuxiang Li, and Aimin Song, Senior \\ Member, IEEE
}

\begin{abstract}
Amorphous indium-gallium-zinc oxide (IGZO) is one of the most promising oxide semiconductors for thin-film transistors and it has started to replace amorphous silicon in display drivers. However, attempts to use IGZO in resistive random-access memories (ReRAMs) are still scarce. This work investigates the bipolar resistive switching properties of crossbarReRAM devices based on IGZO thin film. Aluminium bottom electrode and two different top electrodes (i.e. Al and Ag) were tested in the devices. It was discovered that an oxygen plasma treatment on the bottom electrode could significantly improve the surface roughness of the bottom electrode, the on/off ratio, and the switching uniformity. After the oxygen plasma treatment, the endurance of ReRAMs were enhanced. The on/off current ratios reached $\sim 10^{4}$ and $10^{5}$ within 100 endurance cycles for $\mathrm{Al}$ and $\mathrm{Ag}$ top electrode devices, respectively. Furthermore, the ReRAMs memory window remained nearly constant during a retention test of $10^{5} \mathrm{~s}$. The conduction mechanisms of the two device structures were examined using Schottky emission and space-chargelimited-current pictures, and the memory effect was explained by the formation and the interruption of filaments.
\end{abstract}

Index Terms-IGZO, ReRAMs, oxygen plasma, electrode, memory window, retention time

\section{INTRODUCTION}

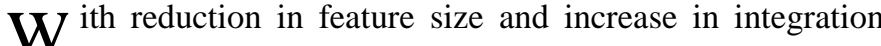
densities, the miniaturisation limit of traditional flash memories is being reached not only because of lithography reasons, but also because of physical limitations, such as large leakage currents[1]. This has prompted all major non-volatile memory manufacturers to seek alternative ways to further increase storage density, such as using three-dimensional (3D) memory arrays[2], [3]. Amongst emerging memory

The authors acknowledge useful discussions with R. Jiang. This work was financed by the National Key Research and Development Program of China (Grant Nos. 2016YFA0201800 and 2016YFA0301200), the National Natural Science Foundation of China (Grant Nos. 11374185, 11304180 and 11374182), Engineering and Physical Sciences Research Council (EPSRC) (Grant No. EP/N021258/1), China Postdoctoral Science Foundation (2015M582073 and 2016M590634), the Natural Science Foundation of Shandong Province (ZR2013EMQ011), the Natural Science Foundation of Jiangsu Province (BK20151255), Suzhou Planning Projects of Science and Technology (SYG201527 and SYG201616), and the Fundamental Research Funds of Shandong University (2013TB008, 2014QY005 and 2016WLJH44).

Pengfei Ma, Guangda Liang, Yiming Wang, Yunpeng Li, Qian Xin, Yuxiang Li, and Aimin Song are with the Center of Nanoelectronics and School of Microelectronics, Shandong University, Jinan 250100, China (email: yxli@sdu.edu.cn).

Aimin Song is also with the School of Electrical and Electronics Engineering, University of Manchester, Manchester M13 9PL, U.K. (e-mail: A.Song@manchester.ac.uk). technologies, resistive random-access memory (ReRAM) devices have significant advantages including high density, stability, high operation speed and low cost[4], [5]. ReRAMs also show promising applications in artificial neural computing fields[6]. The resistance switching behaviour has been investigated in various oxide-based materials, such as $\mathrm{HfO}_{2}[7]-$ [9], $\quad \mathrm{Al}_{2} \mathrm{O}_{3}[10]-[12], \quad \mathrm{Ta}_{2} \mathrm{O}_{5}[13]-[15]$ and $\mathrm{ZnO}[16]-[18]$. Amorphous indium-gallium-zinc oxide (IGZO) is one of the most mature channel materials for thin-film transistors (TFTs) because of its high transparency, flexibility, high carrier mobility and wide range of process temperature[19]-[22]. As such, IGZO TFTs have started replacing amorphous-silicon transistors in backplane drivers display[23], [24]. Our previous work demonstrated that IGZO TFTs are suitable for future wearable and other low-power electronics because of their high yield and low operation voltages[19], [20]. IGZO films have recently been found to exhibit resistance switching properties[25]-[29]. Liu et al. demonstrated an integration of IGZO TFT and ReRAMs to form a one-transistor-one-resistor (1T1R) configuration[30]. Unfortunately, the IGZO-based ReRAMs fabricated at room temperature generally showed a small memory window (i.e. the on/off ratio was mostly between 10 and $\left.10^{2}\right)$ [25]-[29]. Furthermore, multi-level memory devices have been proposed for 3D NAND flash[31], [32]. A sufficiently large memory window is a prerequisite for multilevel storage. Pei et al. showed that inserting a $\mathrm{SiO}_{2}$ buffer layer into IGZO ReRAM devices can improve the resistive switching ratios[33]. Various treatments of IGZO layers have also been made to improve the performance of ReRAMs, e.g., using ultraviolet (UV) irradiation[26], microwave irradiation[34] and element doping[35]. Moreover, the effects of electrode materials on device characteristics have been studied[36], [37]. Inert metals like platinum $(\mathrm{Pt})$ have been used as the ReRAM electrode, especially as the bottom electrode[5], [17], [18], [37].

In this work, high-performance ReRAMs with bipolar resistive switching properties were fabricated using IGZO as the switching layer. The ReRAMs were found to exhibit much improved resistive switching uniformity and endurance characteristics when the oxygen plasma treatment (OPT) was applied to the bottom $\mathrm{Al}$ electrode. Two structures with different top electrodes, namely $\mathrm{Al} / \mathrm{IGZO} / \mathrm{Al}$ and $\mathrm{Ag} / \mathrm{IGZO} / \mathrm{Al}$, are compared. The memory windows reached $10^{5}$ within 100 endurance cycles. Such a switching ratio is orders of magnitude higher than that of the previously reported IGZOReRAMs[25]-[27], [38]-[40]. Furthermore, the memory window of our ReRAMs remains nearly constant during a 
retention test of $10^{5} \mathrm{~s}$. The conduction and switching mechanism was also analysed by examining different possible models.

\section{EXPERIMENT}

The schematic structure of the fabricated ReRAMs is shown in the inset of Fig. 1(a). The process sequence is as follows. A 100-nm Al layer was deposited on a $\mathrm{SiO}_{2} / \mathrm{Si}$ substrate by thermal evaporation to form the bottom electrode. The deposited $\mathrm{Al}$ electrode was subjected to an oxygen plasma treatment for different times $(0 \sim 30 \mathrm{~min})$ at a power of $18 \mathrm{~W}$ to study the influence on the ReRAMs performance. A 30-nm IGZO film (In:Ga:Zn = 1:1:1) was then deposited onto the $\mathrm{Al}$ bottom electrode by radio frequency (RF) sputtering at room temperature. The mixed ambient $\left(\mathrm{Ar}: \mathrm{O}_{2}=18: 3 \mathrm{sccm}\right)$ pressure during sputtering was $3.58 \mathrm{mTorr}$, and the RF power was $90 \mathrm{~W}$. Finally, a $100-\mathrm{nm}$-thick $\mathrm{Al}$ or Ag top electrode was deposited using thermal evaporation. The structures were patterned by photo-lithography methods, and the device active area is $50 \times$ $50 \mu \mathrm{m}^{2}$. The electric characteristics were measured using Agilent B2900. During tests, the bias voltage was applied to the top electrode, whilst the bottom electrode was grounded. The topography and surface roughness of the Al bottom electrode before and after the oxygen plasma treatment were measured using atomic force microscopy (AFM).

\section{RESULTS AND DISCUSSION}
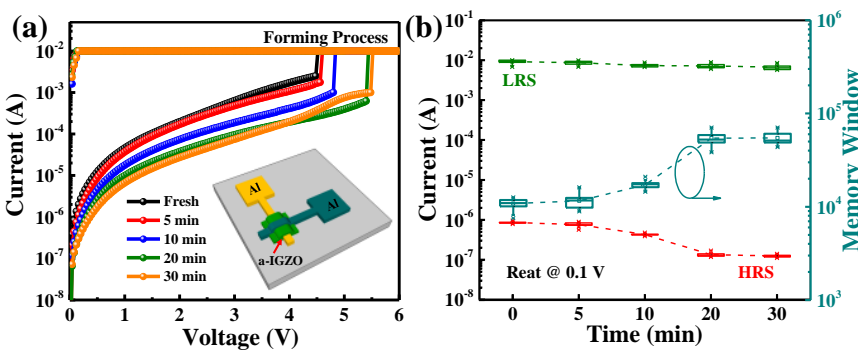

Fig. 1. (a) Forming processes (main graph) and schematic structure (inset) of Al-top IGZO ReRAMs. (b) HRS, LRS and the memory window as a function of the OPT time.

Figure 1(a) shows the forming processes of Al-top ReRAMs with different oxygen plasma treatment times. The initial state of the Al-top devices was all high resistance state (HRS). When a positive bias voltage applied to the top electrode reached a threshold, the device switched from HRS to low resistance state (LRS) drastically. A compliance current $\left(I_{\mathrm{CC}}\right)$ of $10 \mathrm{~mA}$ was set during the forming process, which prevented the devices from unrecoverable breakdown. After the forming process, HRS and LRS could be achieved in turn under bipolar sweep voltages. HRS, LRS and the memory window of the Al-top devices were studied as a function of the oxygen plasma treatment time in Fig. 1(b). With increasing of treatment time, currents of HRS and LRS (measured at $0.1 \mathrm{~V}$ ) gradually decreased from $85.3 \mu \mathrm{A}$ and $9.2 \mathrm{~mA}$ to $12.4 \mu \mathrm{A}$ and $6.7 \mathrm{~mA}$, respectively, and the memory window increased by about two orders of magnitude.
After 20-min oxygen plasma treatment, HRS and LRS no longer improved further and the memory window kept at $5.4 \times$ $10^{4}$. So, we chose $20 \mathrm{~min}$ as the optimum treatment time. After the oxygen plasma treatment, the aluminium surface was oxidised to $\mathrm{Al}_{2} \mathrm{O}_{3}$, which increased the resistance of devices and greatly modified the initial HRS[4].
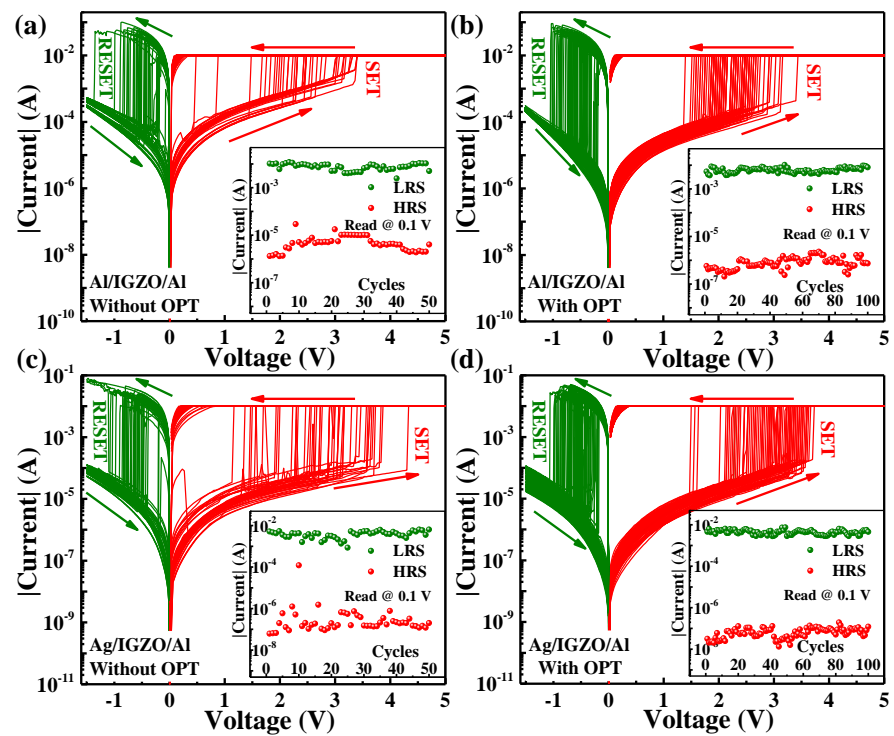

Fig. 2. Typical bipolar resistive switching $I-V$ curves of Al-top devices (a) without or (b) with OPT. Typical bipolar resistive switching $I-V$ curves of $\mathrm{Ag}-$ top devices (c) without or (d) with OPT. The insets show endurance performance of the Al-top and Ag-top devices without and with OPT, in which the current was recorded at $0.1 \mathrm{~V}$.

The endurance characteristic is critical for ReRAMs. Figures 2(a)-(d) show the typical bipolar resistance switching characteristics of Al-top and Ag-top devices after forming processes, respectively. Under positive voltages, the ReRAMs abruptly switched from HRS to LRS, which is defined as the set process. The set voltage is as usual smaller than the forming voltage. The reset process was observed when the resistance was switched from LRS to HRS in the negative voltage region, and hence a non-volatile bipolar resistive switching was achieved. Figure 2 shows a pronounced performance contrast between ReRAMs with and without the oxygen plasma treatment. The devices with the oxygen plasma treatment exhibit not only much higher on/off ratios but also excellent endurance where the resistance switching behaviour can be repeated for more than 100 cycles without obvious degradation. However, the devices without the oxygen plasma treatment showed abnormal cycling curves during the endurance test of 50 cycles, as shown in Figs. 2(a) and 2(c). After 50-cycles test, the devices were degraded (not shown here). The distribution of set voltages for devices with the oxygen plasma treatment was also much narrower than that of non-oxygen-plasmatreatment devices. The insets in Fig. 2 show the detailed evolution of LRS and HRS for devices with and without the oxygen plasma treatment in repeated cycles. The ReRAMs with the oxygen plasma treatment showed a seven times larger 
memory window than devices without the oxygen plasma treatment. The variation of HRS and LRS during the cycling was much smaller for devices with the oxygen plasma treatment, and the memory window maintained consistently at $10^{4}$ and $10^{5}$ for the Al-top and Ag-top devices, respectively. (a)

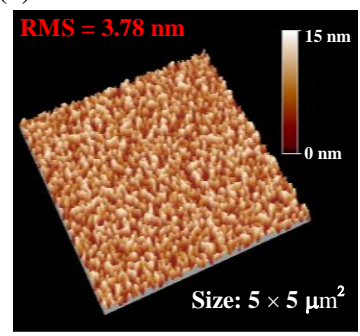

(b)

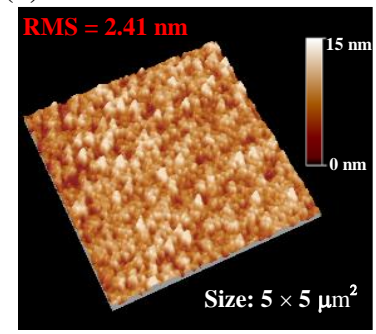

Fig. 3. AFM images of the Al bottom electrode (a) before and (b) after OPT.

The physical property and smoothness of the electrodes is known to plays an important role in improving the performances of ReRAMs[41]. Figures 3(a) and 3(b) show the AFM images of the bottom $\mathrm{Al}$ electrodes before and after the oxygen plasma treatment, respectively. The oxygen plasma treatment enabled the root-mean-square (RMS) surface roughness to decrease from 3.78 to $2.41 \mathrm{~nm}$. Molina et al. found that devices with low surface roughness on the bottom electrode showed a higher number of resistance switching cycles[41]. It was also reported that ReRAMs with superior surface morphology show a better on-off current ratio[42]. The performance of the devices may benefit from the diminution roughness of the $\mathrm{Al}$ bottom electrode by improving electric fields for breakdown during resistance switching sweeps[41]. Furthermore, a thin but dense layer of $\mathrm{Al}_{2} \mathrm{O}_{3}$ is expected to form on the aluminium electrode surface after the oxygen plasma treatment[43]. It was found that an $\mathrm{Al}_{2} \mathrm{O}_{3}$ buffer layer played an important role to improve the resistance ratios in $\mathrm{ZnO}-$ ReRAMs[44], and the endurance characteristics of $\mathrm{Ta}_{5} \mathrm{Si}_{3}-$ ReRAMs[45].

To better analyse the performance of ReRAMs, Figs. 4(a)(d) illustrate the distributions of the threshold voltages (set and reset voltages) for Al-top and Ag-top memory devices without and with the oxygen plasma treatment, respectively. The black solid lines represent the Gaussian fitting results. The $N\left(\mu, \sigma^{2}\right)$ values of four types of samples are summarised in Table I, where $\mu$ is the mean value of set voltage $\left(V_{\text {set }}\right)$ or reset voltage ( $\left.V_{\text {reset }}\right)$, and $\sigma$ is the standard deviation. As shown in Table I, $V_{\text {reset }}$ is smaller than $V_{\text {set }}$ for both types of devices. This is expected because the reset process occurs at a higher current and the Joule heating may facilitate the rupture of filaments[40]. It can be found that after the oxygen plasma treatment, the distribution of $V_{\text {set }}$ is decreased for both types of devices. For Al-top devices, $\sigma$ decreased from $0.8 \mathrm{~V}$ to $0.4 \mathrm{~V}$, and for Agtop devices, $\sigma$ decreased from $0.7 \mathrm{~V}$ to $0.5 \mathrm{~V}$. The distribution of $V_{\text {set }}$ is wide for devices without the oxygen plasma treatment, suggesting that the formation of filaments randomly occurred because of the irregular surface of the bottom electrodes. During the reset process, the breakage of filaments usually occurs inside the resistance switching layer and is little associated with the bottom electrode surface[46]. So, the $\sigma$ values of $V_{\text {reset }}$ are about the same for both types of ReRAMs as shown in Table I.
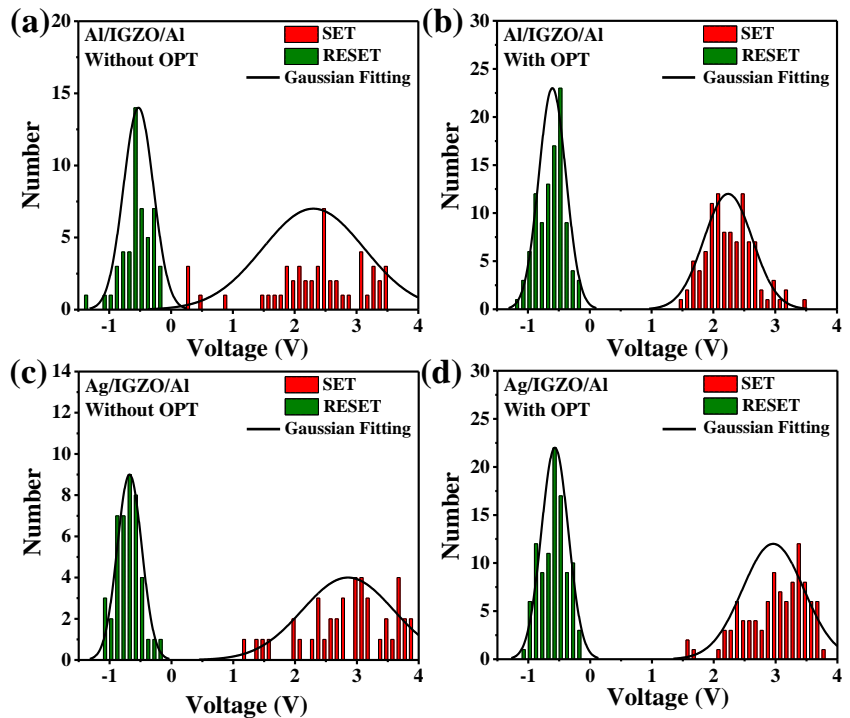

Fig. 4. Distributions of the threshold voltages of the Al-top devices (a) without or (b) with OPT. Distributions of the threshold voltages of the Ag-top devices (c) without or (d) with OPT. The solid lines are the Gaussian fittings of the distributions.

TABLE I

Comparison of $\mathrm{N}\left(\mu, \sigma^{2}\right)$ values of the four types of IGZO-ReRAMs

\begin{tabular}{ccccc}
\hline \hline Devices & $\begin{array}{c}\boldsymbol{\mu} \text { of } \boldsymbol{V}_{\text {set }} \\
(\mathbf{V})\end{array}$ & $\begin{array}{c}\boldsymbol{\sigma} \text { of } \boldsymbol{V}_{\text {set }} \\
(\mathbf{V})\end{array}$ & $\begin{array}{c}\boldsymbol{\mu} \text { of } \boldsymbol{V}_{\text {reset }} \\
(\mathbf{V})\end{array}$ & $\begin{array}{c}\boldsymbol{\sigma} \text { of } \boldsymbol{V}_{\text {reset }} \\
(\mathbf{V})\end{array}$ \\
\hline $\begin{array}{c}\text { Al-top devices } \\
\text { without OPT }\end{array}$ & 2.3 & 0.8 & -0.5 & 0.2 \\
$\begin{array}{c}\text { Al-top devices } \\
\text { with OPT } \\
\begin{array}{c}\text { Ag-top devices } \\
\text { without OPT }\end{array}\end{array}$ & 2.2 & 0.4 & -0.6 & 0.2 \\
$\begin{array}{c}\text { Ag-top devices } \\
\text { with OPT }\end{array}$ & 3.0 & 0.7 & -0.7 & 0.2 \\
\hline \hline
\end{tabular}

The retention performance of our IGZO-ReRAMs without and with the oxygen plasma treatment at room temperature is illustrated in Fig. 5. The on and off currents remained nearly constant for all devices during a retention test up to $10^{5} \mathrm{~s}$, and the information storage in our devices could be maintained for a much longer time (10 years) according to the trend of data showing by the dashed lines in Fig. 5. This confirms the nonvolatile characteristics of our devices. In Table II, the key parameters of our devices are compared with those of the previously reported IGZO-ReRAMs devices. Most of IGZOReRAMs and our devices showed bipolar switching type, which means the set and reset process occur at different voltage polarities. Unipolar type means that the switching direction doesn't depend on the voltage polarity. The switching type usually depends on the electrodes and fabrication process[47]. On/off ratios $>10$ are required in sensing amplifiers [46]. While for previous reports, the on/off ratios of IGZO-ReRAMs were 
mostly between 10 and $10^{2}$. Table II shows on/off ratios of $10^{4}$, $10^{5}$ in Our IGZO-ReRAMs, which are high enough to enable multi-level memory applications[27]. Our devices also showed high endurance and durability characteristics as compared with the previous works.
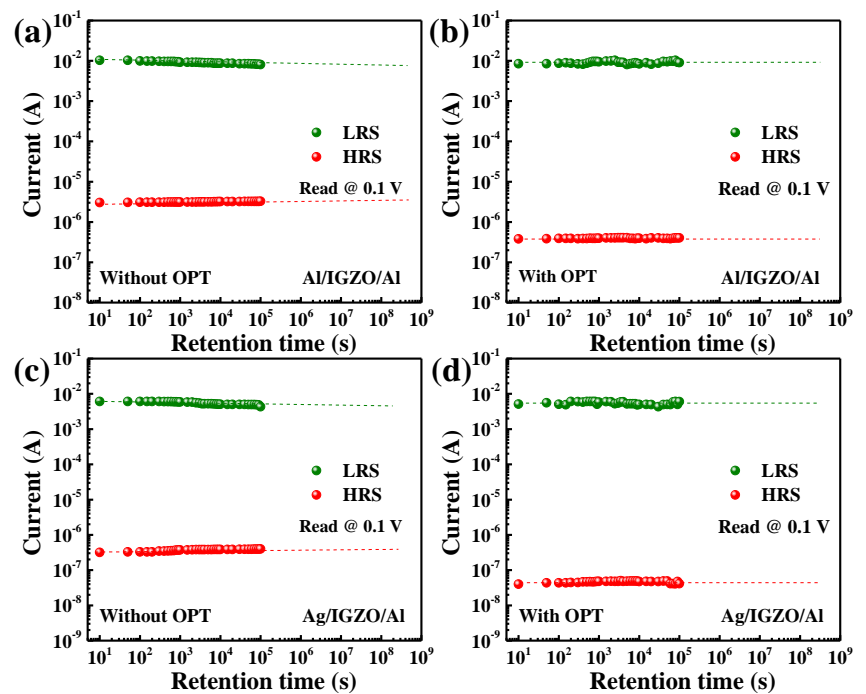

Fig. 5. Retention performances of (a) Al-top devices (a) without or (b) with OPT, and Ag-top devices (c) without or (d) with OPT. The HRS and LRS current values were recorded at $0.1 \mathrm{~V}$.

TABLE II

Comparison of the performance parameters of the IGZO-ReRAMs with Al and $\mathrm{Ag}$ electrodes and with the oxygen plasma treatment.

\begin{tabular}{ccccc}
\hline \hline Structures & Type & $\begin{array}{c}\text { On/off } \\
\text { ratios }\end{array}$ & Cycles & $\begin{array}{c}\text { Retention } \\
\text { (s) }\end{array}$ \\
\hline $\mathrm{ITO} / \mathrm{IGZO} / \mathrm{ITO}[38]$ & Bipolar & $\sim 10$ & 100 & $10^{4}$ \\
$\mathrm{Ti} / \mathrm{IGZO} / \mathrm{Pt}[39]$ & Bipolar & $\sim 10$ & 100 & $10^{4}$ \\
$\mathrm{Ag} / \mathrm{IGZO} / \mathrm{Pt}[25]$ & Bipolar & $\sim 10^{2}$ & 100 & $10^{4}$ \\
$\mathrm{Pt} / \mathrm{IGZO} / \mathrm{Pt}[26]$ & Bipolar & $\sim 10^{2}$ & $/$ & $10^{5}$ \\
$\mathrm{Ti} / \mathrm{IGZO} / \mathrm{Pt}[27]$ & Bipolar & $\sim 10^{2}$ & 150 & $10^{3}$ \\
$\mathrm{Cu} / \mathrm{IGZO} / \mathrm{Cu}[40]$ & Unipolar & $\sim 10^{2}$ & 150 & $10^{5}$ \\
$\mathrm{Ti} / \mathrm{IGZO} / \mathrm{Pt}[34]$ & Bipolar & $\sim 10^{2}$ & 100 & $10^{4}$ \\
$\mathrm{Ag} / \mathrm{SiO} / / \mathrm{IGZO} / \mathrm{Pt}[33]$ & Bipolar & $\sim 10^{4}$ & 100 & $10^{4}$ \\
\hline $\mathbf{T h h i s ~ A l / I G Z O / A l}$ & Bipolar & $\sim \mathbf{1 0}^{4}$ & $\mathbf{1 0 0}$ & $\mathbf{1 0}^{5}$ \\
work) Ag/IGZO/Al & Bipolar & $\sim \mathbf{1 0}^{5}$ & $\mathbf{1 0 0}$ & $\mathbf{1 0}^{5}$ \\
\hline \hline
\end{tabular}

The uniformity of our devices with the oxygen plasma treatment was also tested. The forming processes of 25 devices for both $\mathrm{Al}$ and $\mathrm{Ag}$ top electrode cases are shown in Fig. 6(a). Excellent uniformity was clearly achieved. The forming voltage differs by $1.8 \%$ and $2.4 \%$, respectively. The initial resistance of the Ag-top ReRAMs was larger than that of the Al-top devices, which may be caused by the different metal work functions. Figures 6(e) and 6(f) show schematic energy band diagrams of the Al-top and Ag-top structures, respectively. The work function values of $\mathrm{Ag}, \mathrm{Al}$ and $\mathrm{IGZO}$ were $4.52, \sim 4.06$ and $\sim 4.36 \mathrm{eV}$, respectively[48]. Thus, the metal/semiconductor contacts of $\mathrm{Ag} / \mathrm{IGZO}$ and $\mathrm{Al} / \mathrm{IGZO}$ are Schottky and Ohmic types, respectively. It is difficult for carriers to cross the Schottky barrier between Ag and IGZO, which leads to a lower current.

To study the vertical carrier transport mechanism, we fitted the $I-V$ curves with different models, including Ohmic,
Schottky emission, Poole-Frenkel (P-F) emission, trapassisted tunnelling and space-charge-limited-current (SCLC). These mechanisms are generally observed in ReRAMs[49]. The SCLC in the Child's square law region can be expressed as:

$$
J=\frac{9 \varepsilon_{\mathrm{s}} \mu V^{2}}{8 d^{3}}
$$

where $J$ is the current density, $\varepsilon_{\mathrm{s}}$ is the semiconductor permittivity, $\mu$ is the drift mobility, $V$ is the bias voltage, and $d$ is the semiconductor thickness[49]. For the Schottky emission, the $I-V$ characteristic is as follow:

$$
I \propto T^{2} \exp \left[\frac{q}{k T}\left(a \sqrt{V}-\phi_{\mathrm{B}}\right)\right]
$$

where $T$ is the absolute temperature, $k$ is the Boltzmann constant, $q$ is the electron charge, $a$ is a constant, and $\phi_{\mathrm{B}}$ is the barrier height[49].


Fig. 6. (a) Forming process of IGZO-ReRAMs of two structures with OPT. Double-logarithmic $I-V$ plots of the (b) Al-top and (c) Ag-top devices. (d) Fitting line with Schottky emission mechanism for the HRS region of the $\mathrm{Ag}-$ top devices. Energy band diagrams of the (e) Al-top and (f) Ag-top devices.

Figures 6(b) and 6(c) show double-logarithmic $I-V$ plots of the Al-top and Ag-top devices, respectively. The dotted lines are the experimented results, and the solid lines are the fittings. Based on the fittings, a few conclusions can be drawn. For the Al-top devices, a typical SCLC model fits well in the HRS region, which consists of three portions[49]. In the low bias voltage regime, the thermally generated carriers played the leading roles. The current follows Ohmic behaviour with a slope of $\sim 1$, as shown in Fig. 6(b). The Child's law region $(I \propto$ $V^{2}$ ) with a slope of $\sim 2$ is known as the trap-unfilled SCLC region. By increasing the applied bias voltage, more carriers deep entered the IGZO resistance switching layer. The traps 
within the resistance switching layer were filled with the carriers, leading to the steep current increase region. For the Ag-top devices, Fig. 6(d) shows a good linear fitting of the HRS region by Schottky emission according to Eq. (2), which agrees with the model in Fig 6(c). Other conduction models do not provide such a good agreement, indicating that the Schottky emission should be the vertical transport mechanism for the Ag-top devices in the HRS. As for the LRS, the linear fittings showed that the $I-V$ relationships comply with the Ohmic conduction behaviour with a slope of $\sim 1$ for both the structure devices, which usually suggesting formation of conductive filaments in the ReRAMs[5].

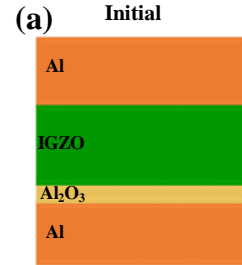

(b)
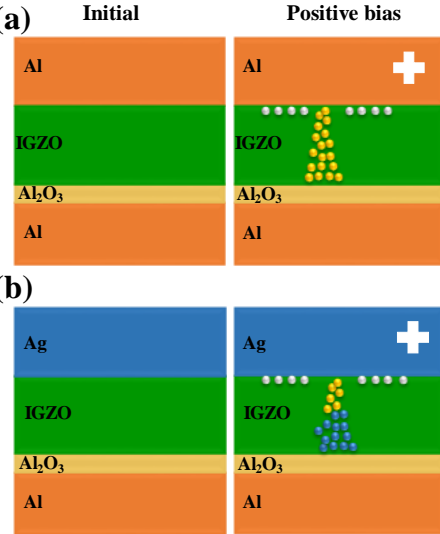

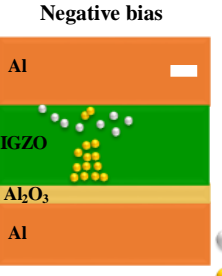

Oxygen ion Oxygen vacancy

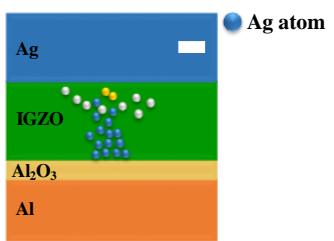

Fig. 7. Schematic illustration of the resistance switching mechanisms of the (a) Al-top and (b) Ag-top devices with OPT.

To understand the pronounced improvement of the oxygen plasma treatment on Al-bottom electrode, schematics based on the filament formation and rupture are illustrated in Fig. 7. The thin $\mathrm{Al}_{2} \mathrm{O}_{3}$ layer is shown on the $\mathrm{Al}$ bottom electrode. For the Al-top ReRAMs in Fig. 7(a), the Al-top electrode would attract oxygen ions from the IGZO layer under a positive bias voltage. Meanwhile, oxygen vacancies can accumulate near the bottom electrode, and a conductive filament is gradually formed to connect the top and bottom electrodes. For the Ag-top ReRAMs in Fig. 7(b), when a positive bias voltage is applied to the top electrode, $\mathrm{Ag}$ can oxidise to $\mathrm{Ag}^{+}\left(\mathrm{Ag} \rightarrow \mathrm{Ag}^{+}+\mathrm{e}^{-}\right)$by the mobile oxygen from weakly-bonded oxygen defects[25]. The mobile $\mathrm{Ag}^{+}$cations can pass through the IGZO layer and move to the bottom electrode because of the small radius and the high mobility of ions[5]. The reduction reaction of the $\mathrm{Ag}^{+}$ cations $\left(\mathrm{Ag}^{+}+\mathrm{e}^{-} \rightarrow \mathrm{Ag}\right)$ then occurs when the electrons pass through the cathode[18]. The successive formation of the $\mathrm{Ag}$ atoms at the bottom electrode results in a growth of the $\mathrm{Ag}$ filament. Meanwhile, the oxygen ions are attracted to the top electrode, following the formation of the oxygen vacancy filament in the IGZO layer[25]. Moreover, the Ag filament formed close to the bottom electrode may contribute to a stronger electric field at the filament tip. The oxygen vacancy filament is easy to form and connect with the tip of the $\mathrm{Ag}$ filament, which may lead to the smaller forming voltage for the Ag-top devices, as shown in Fig. 6(a). During the reset process, the oxygen ions can be released from the top electrode under the negative bias voltage. These ions may move back to the IGZO film and neutralise oxygen vacancies, leading to a breakage of the filaments and hence the HRS[35].


Fig. 8. (a) HRS and LRS currents versus size of the (a) Al-top and (b) Ag-top ReRAMs with OPT. The currents were recorded at $0.1 \mathrm{~V}$.

The ReRAMs with the oxygen plasma treatment were also examined with different active areas to verify the filament model. Figure 8 shows the relationship of the HRS and LRS currents with various active device sizes. The currents of the HRS were in direct proportion to the active areas for the two structures (clearer in linear scale, not shown here), as expected with the Ohm law. In contrast, the LRS currents only showed a slight dependence on the active area, as expected with filamentary conduction. The finding suggests that the memory window may be further improved by scaling.

\section{CONCLUSIONS}

In summary, IGZO-ReRAMs are demonstrated with excellent bipolar resistance switching characteristics. We found that a suitable oxygen plasma treatment can reduce the surface roughness of the bottom $\mathrm{Al}$ electrode and generate an $\mathrm{Al}_{2} \mathrm{O}_{3}$ buffer layer, which improves the ReRAM performance significantly. Importantly, a memory window up to $10^{5}$ remains quite stable in 100 endurance cycles and for a retention time of $10^{5} \mathrm{~s}$. The conduction mechanism of the two device structures with different top electrodes has been analysed by fitting with different models. The formation and interrupt of conductive filaments is proposed to lead to the memory effect. Since IGZO has already been adopted in the display industry for thin-film transistors, the addition of IGZO-based high performance nonvolatile ReRAMs could enable more functional oxide-based circuits for a range of applications such as wearable electrons and smart lables.

\section{REFERENCES}

[1] F. Pan, S. Gao, C. Chen, C. Song, and F. Zeng, "Recent Rrogress in Resistive Random Access Memories: Materials, Switching Mechanisms, and Performance," Mater. Sci. Eng., R, vol. 83, pp. 1-59, Sep 2014. doi: 10.1016/j.mser.2014.06.002

[2] H. Tanaka, M. Kido, K. Yahashi, M. Oomura, R. Katsumata, M. Kito, Y. Fukuzumi, M. Sato, Y. Nagata, Y. Matsuoka, Y. Iwata, H. Aochi, A. Nitayama, and P. Japan Soc Appl, "Bit Cost Scalable Technology with Punch and Plug Pprocess for Ultra High Density Flash Memory," IEEE Symp. VLSI Technol., pp. 14-15, 2007. doi: 10.1109/vlsit.2007.4339708

[3] K.-T. Park, S. Nam, D. Kim, P. Kwak, D. Lee, Y.-H. Choi, M.-H. Choi, D.H. Kwak, D.-H. Kim, M.-S. Kim, H.-W. Park, S.-W. Shim, K.-M. Kang, S.-W. Park, K. Lee, H.-J. Yoon, K. Ko, D.-K. Shim, Y.-L. Ahn, J. Ryu, D. 
Kim, K. Yun, J. Kwon, S. Shin, D.-S. Byeon, K. Choi, J.-M. Han, K.-H. Kyung, J.-H. Choi, and K. Kim, "Three-Dimensional 128 Gb MLC Vertical NAND Flash Memory With 24-WL Stacked Layers and $50 \mathrm{MB} / \mathrm{s}$ HighSpeed Programming," IEEE J. Solid-State Circuits, vol. 50, no. 1, pp. $204-$ 213, Jan 2015. doi: 10.1109/jssc.2014.2352293

[4] J.-G. Park, W.-S. Nam, S.-H. Seo, Y.-G. Kim, Y.-H. Oh, G.-S. Lee, and U.G. Paik, "Multilevel Nonvolatile Small-Molecule Memory Cell Embedded with Ni Nanocrystals Surrounded by a NiO Tunneling Barrier," Nano Lett., vol. 9, no. 4, pp. 1713-1719, Apr 2009. doi: 10.1021/n1900429h

[5] C.-F. Chang, J.-Y. Chen, C.-W. Huang, C.-H. Chiu, T.-Y. Lin, P.-H. Yeh, and W.-W. Wu, "Direct Observation of Dual-Filament Switching Behaviors in $\mathrm{Ta}_{2} \mathrm{O}_{5}$-Based Memristors," Small, vol. 13, no. 15, p. 1603116, 2017-Apr 2017. doi: 10.1002/smll.201603116

[6] G. W. Burr, R. M. Shelby, A. Sebastian, S. Kim, S. Kim, S. Sidler, K. Virwani, M. Ishii, P. Narayanan, A. Fumarola, L. L. Sanches, I. Boybat, M. Le Gallo, K. Moon, J. Woo, H. Hwang, and Y. Leblebici, "Neuromorphic Computing Using Non-Volatile Memory," Adv. Phys., vol. 2, no. 1, pp. 89124, 2017 2017. doi: 10.1080/23746149.2016.1259585

[7] G. Niu, P. Calka, M. A. der Maur, F. Santoni, S. Guha, M. Fraschke, P. Hamoumou, B. Gautier, E. Perez, C. Walczyk, C. Wenger, A. Di Carlo, L. Alff, and T. Schroeder, "Geometric Conductive Filament Confinement by Nanotips for Resistive Switching of $\mathrm{HfO}_{2}$-RRAM Devices with High Performance," Sci. Rep., Article vol. 6, p. 9, May 2016. doi: $10.1038 /$ srep25757

[8] J. Shang, G. Liu, H. Yang, X. Zhu, X. Chen, H. Tan, B. Hu, L. Pan, W. Xue, and R.-W. Li, "Thermally Stable Transparent Resistive Random Access Memory based on All-Oxide Heterostructures," Adv. Funct. Mater., vol. 24, no. 15, pp. 2171-2179, Apr 2014. doi: 10.1002/adfm.201303274

[9] H. Borkar, A. Thakre, S. S. Kushvaha, R. P. Aloysius, and A. Kumar, "Light Assisted Irreversible Resistive Switching in Ultra Thin Hafnium Oxide," RSC Adv., vol. 5, no. 44, pp. 35046-35051, 2015 2015. doi: $10.1039 / \mathrm{c} 5 \mathrm{ra03352 \textrm {g }}$

[10] S. W. Yeom, S. C. Shin, T. Y. Kim, H. J. Ha, Y. H. Lee, J. W. Shim, and B. K. Ju, "Transparent Resistive Switching Memory Using Aluminum Oxide on a Flexible Substrate," Nanotechnology, Article vol. 27, no. 7, p. 6, Feb 2016. doi: 10.1088/0957-4484/27/7/071t01

[11] Q. H. Li, L. J. Qiu, X. H. Wei, B. Dai, and H. Z. Zeng, "Point Contact Resistive Switching Memory Based on Self-Formed Interface of Al/ITO," Sci. Rep., Article vol. 6, p. 8, Jul 2016. doi: 10.1038/srep29347

[12] S. Kim and B.-G. Park, "Nonlinear and Multilevel Resistive Switching Memory in $\mathrm{Ni} / \mathrm{Si}_{3} \mathrm{~N}_{4} / \mathrm{Al}_{2} \mathrm{O}_{3} / \mathrm{TiN}$ Structures," Appl. Phys. Lett., vol. 108, no. 21, p. 212103, 2016. doi: 10.1063/1.4952719

[13] X. Y. Li, H. Q. Wu, B. Gao, W. Wu, D. Wu, N. Deng, J. Cai, and H. Qian, "Electrode-Induced Digital-to-Analog Resistive Switching in $\mathrm{TaO}_{\mathrm{x}}$-Based RRAM Devices," Nanotechnology, Article vol. 27, no. 30, p. 6, Jul 2016. doi: 10.1088/0957-4484/27/30/305201

[14] X. Chen, J. Feng, and D. Bae, "Drastic Reduction of RRAM Reset Current Via Plasma Oxidization of TaOx Film," Appl. Surf. Sci., vol. 324, pp. 275279, Jan 1 2015. doi: 10.1016/j.apsusc.2014.10.133

[15] V. Y. Q. Zhuo, M. Li, Y. Guo, W. Wang, Y. Yang, Y. Jiang, and J. Robertson, "CMOS Compatible Electrode Materials Selection in OxideBased Memory Devices," J. Appl. Phys., Article vol. 120, no. 2, p. 7, Jul 2016. doi: 10.1063/1.4955044

[16] F. C. Chiu, P. W. Li, and W. Y. Chang, "Reliability Characteristics and Conduction Mechanisms in Resistive Switching Memory Devices Using ZnO Thin Films," Nanoscale Res. Lett., vol. 7, p. 178, Mar 8 2012. doi: 10.1186/1556-276x-7-178

[17] F. M. Simanjuntak, D. Panda, K.-H. Wei, and T.-Y. Tseng, "Status and Prospects of ZnO-Based Resistive Switching Memory Devices," Nanoscale Res. Lett., vol. 11, p. 368, Aug 19 2016. doi: 10.1186/s11671016-1570-y

[18] Y. C. Yang, F. Pan, Q. Liu, M. Liu, and F. Zeng, "Fully RoomTemperature-Fabricated Nonvolatile Resistive Memory for Ultrafast and High-Density Memory Application," Nano Lett., vol. 9, no. 4, pp. 16361643, Apr 2009. doi: 10.1021/n1900006g

[19] P. Ma, L. Du, Y. Wang, R. Jiang, Q. Xin, Y. Li, and A. Song, "Low Voltage Operation of IGZO Thin Film Transistors Enabled by Ultrathin $\mathrm{Al}_{2} \mathrm{O}_{3}$ Gate Dielectric," Appl. Phys. Lett., vol. 112, no. 2, p. 023501, Jan 8 2018. doi: $10.1063 / 1.5003662$

[20] P. Ma, J. Sun, G. Liang, Y. Li, Q. Xin, Y. Li, and A. Song, "Half-volt Operation of IGZO Thin-Film Transistors Enabled by Ultrathin $\mathrm{HfO}_{2}$ Gate Dielectric," Appl. Phys. Lett., vol. 113, no. 6, p. 063501, Aug 6 2018. doi: $10.1063 / 1.5037410$

[21] K. Nomura, H. Ohta, A. Takagi, T. Kamiya, M. Hirano, and H. Hosono, "Room-Temperature Fabrication of Transparent Flexible Thin-Film
Transistors Using Amorphous Oxide Semiconductors," Nature, vol. 432, no. 7016, pp. 488-492, Nov 25 2004. doi: 10.1038/nature03090

[22] S. Lee and A. Nathan, "Subthreshold Schottky-Barrier Thin-Film Transistors with Ultralow Power and High Intrinsic Gain," Science, vol. 354, no. 6310, pp. 302-304, Oct 21 2016. doi: 10.1126/science.aah5035

[23] S. Nakano, N. Saito, K. Miura, T. Sakano, T. Ueda, K. Sugi, H. Yamaguchi, I. Amemiya, M. Hiramatsu, and A. Ishida, "Highly Reliable a-IGZO TFTs on a Plastic Substrate For Flexible AMOLED Displays," J. Soc. Inf. Disp., vol. 20, no. 9, pp. 493-498, Sep 2012. doi: 10.1002/jsid.111

[24] J.-h. Lee, D.-h. Kim, D.-j. Yang, S.-y. Hong, K.-s. Yoon, P.-s. Hong, C.-o. Jeong, H.-s. Park, S. Y. Kim, S. K. Lim, S. S. Kim, K.-s. Son, T.-s. Kim, J.-y. Kwon, S.-y. Lee, and Sid, "World's Largest (15-inch) XGA AMLCD Panel Using IGZO Oxide TFT," SID Symp. Dig. Tech. Pap., vol. 39, pp. 625-628, 2008. doi: 10.1889/1.3069740

[25] Y. L. Pei, B. R. Mai, X. K. Zhang, R. Q. Hu, Y. Li, Z. M. Chen, B. F. Fan, J. Liang, and G. Wang, "Forming Free Bipolar ReRAM of Ag/a-IGZO/Pt with Improved Resistive Switching Uniformity Through Controlling Oxygen Partial Pressure," J. Electron. Mater., Article vol. 44, no. 2, pp. 645-650, Feb 2015. doi: 10.1007/s11664-014-3547-x

[26] W. Hu, L. L. Zou, X. M. Chen, N. Qin, S. W. Li, and D. H. Bao, "Highly Uniform Resistive Switching Properties of Amorphous InGaZnO Thin Films Prepared by a Low Temperature Photochemical Solution Deposition Method," ACS Appl. Mater. Interfaces, Article vol. 6, no. 7, pp. 5012-5017, Apr 2014. doi: 10.1021/am500048y

[27] C.-H. Hsu, Y.-S. Fan, and P.-T. Liu, "Multilevel Resistive Switching Memory with Amorphous InGaZnO-Based Thin Film," Appl. Phys. Lett., vol. 102, no. 6, p. 062905, 2013. doi: 10.1063/1.4792316

[28] X. B. Yan, H. Hao, Y. F. Chen, Y. C. Li, and W. Banerjee, "Highly Transparent Bipolar Resistive Switching Memory with In-Ga-Zn-O Semiconducting Electrode in In-Ga-Zn-O/ $\mathrm{Ga}_{2} \mathrm{O}_{3} / \mathrm{In}-\mathrm{Ga}-\mathrm{Zn}-\mathrm{O}$ Structure," Appl. Phys. Lett., vol. 105, no. 9, p. 093502, Sep 1 2014. doi: $10.1063 / 1.4894521$

[29] M.-S. Kim, Y. Hwan Hwang, S. Kim, Z. Guo, D.-I. Moon, J.-M. Choi, M.L. Seol, B.-S. Bae, and Y.-K. Choi, "Effects of The Oxygen Vacancy Concentration in InGaZnO-Based Resistance Random Access Memory," Appl. Phys. Lett., vol. 101, no. 24, p. 243503, 2012. doi: $10.1063 / 1.4770073$

[30] C.-C. Chang, P.-T. Liu, C.-Y. Chien, and Y.-S. Fan, "Solving the Integration Problem of One Transistor One Memristor Architecture with a Bi-layer IGZO Film Through Synchronous Process," Appl. Phys. Lett., vol. 112, no. 17, p. 172101, Apr 23 2018. doi: 10.1063/1.5020583

[31] K. Mizoguchi, T. Takahashi, S. Aritome, and K. Takeuchi, "DataRetention Characteristics Comparison of 2D and 3D TLC NAND Flash Memories," IEEE IMW, pp. 1-4, 2017. doi: 10.1109/imw.2017.7939077

[32] K. Ho-Jung, C. Nagyong, L. Dong Hwan, L. Tackhwi, C. Sungyong, B. Jong-Ho, P. Byung-Gook, and L. Jong-Ho, "Space Program Scheme for 3D NAND Flash Memory Specialized for the TLC Design," IEEE Symp. VLSI Technol., pp. 201-2, 2018. doi: 10.1109/vlsit.2018.8510660

[33] Y. L. Pei, B. R. Mai, X. K. Zhang, R. Q. Hu, Y. Li, Z. M. Chen, B. F. Fan, J. Liang, and G. Wang, "Performance Improvement of Amorphous IndiumGallium-Zinc Oxide ReRAM with $\mathrm{SiO}_{2}$ Inserting Layer," Curr. Appl. Phys., Article vol. 15, no. 4, pp. 441-445, Apr 2015. doi: 10.1016/j.cap.2015.01.024

[34] Y. H. Hwang, H. M. An, and W. J. Cho, "Performance Improvement of the Resistive Memory Properties of InGaZnO Thin Films by Using Microwave Irradiation," Jpn. J. Appl. Phys., Article vol. 53, no. 4, p. 4, Apr 2014. doi: 10.7567/jjap.53.04ej04

[35] Q. Li, Y. H. Li, L. W. Gao, F. Ma, Z. X. Song, and K. W. Xu, "Ru Doping Enhanced Resistive Switching Behavior in InGaZnO Thin Films," RSC Adv., Article vol. 6, no. 48, pp. 42347-42352, 2016. doi: $10.1039 / \mathrm{c} 6 \mathrm{ra} 02174 \mathrm{c}$

[36] Y.-J. Huang, T.-H. Shen, L.-H. Lee, C.-Y. Wen, and S.-C. Lee, "LowPower Resistive Random Access Memory by Confining the Formation of Conducting Filaments," AIP Adv., vol. 6, no. 6, p. 065022, Jun 2016. doi: $10.1063 / 1.4954974$

[37] X. Wang, H. Qian, L. Guan, W. Wang, B. Xing, X. Yan, S. Zhang, J. Sha, and Y. Wang, "Influence of Metal Electrode on the Performance of $\mathrm{ZnO}$ Based Resistance Switching Memories," J. Appl. Phys., vol. 122, no. 15, p. 154301, Oct 21 2017. doi: 10.1063/1.4996975

[38] M.-C. Chen, T.-C. Chang, S.-Y. Huang, S.-C. Chen, C.-W. Hu, C.-T. Tsai, and S. M. Sze, "Bipolar Resistive Switching Characteristics of Transparent Indium Gallium Zinc Oxide Resistive Random Access Memory," Electrochem. Solid-State Lett., vol. 13, no. 6, p. H191, 2010. doi: $10.1149 / 1.3360181$ 
[39] Y. H. Hwang, I. Hwang, and W. J. Cho, "Composition-Ratio Influence on Resistive Switching Behavior of Solution-Processed InGaZnO-Based Thin-Film," J. Nanosci. Nanotechnol., Article vol. 14, no. 11, pp. 81968200, Nov 2014. doi: 10.1166/jnn.2014.9892

[40] Z. Q. Wang, H. Y. Xu, X. H. Li, X. T. Zhang, Y. X. Liu, and Y. C. Liu, "Flexible Resistive Switching Memory Device Based on Amorphous InGaZnO Film With Excellent Mechanical Endurance," IEEE Electron Device Lett., vol. 32, no. 10, pp. 1442-1444, 2011. doi: 10.1109/led.2011.2162311

[41] J. Molina, R. Valderrama, C. Zuniga, P. Rosales, W. Calleja, A. Torres, J. DeLa Hidalga, and E. Gutierrez, "Influence of the Surface Roughness of the Bottom Electrode on the Resistive-Switching Characteristics of $\mathrm{Al} / \mathrm{Al}_{2} \mathrm{O}_{3} / \mathrm{Al}$ and $\mathrm{Al} / \mathrm{Al}_{2} \mathrm{O}_{3} / \mathrm{W}$ Structures Fabricated on Glass at $300^{\circ} \mathrm{C}$," Microelectron. Reliab., vol. 54, no. 12, pp. 2747-2753, 2014. doi: 10.1016/j.microrel.2014.07.006

[42] D. L. Chen, H. C. Yu, C. C. Yang, Y. K. Su, C. W. Chou, and J. L. Ruan, "Performance Enhancement of $\mathrm{Pt} / \mathrm{ZnO} / \mathrm{Pt}$ Resistive Random Access Memory (RRAM) with UV-Ozone Treatment," AM-FPD, pp. 213-214, 2016. doi: 10.1109/AM-FPD.2016.7543670

[43] B. Cho, S. Song, Y. Ji, and T. Lee, "Electrical Characterization of Organic Resistive Memory with Interfacial Oxide Layers Formed by $\mathrm{O}_{2}$ Plasma Treatment," Appl. Phys. Lett., vol. 97, no. 6, p. 063305, Aug 9 2010. doi: $10.1063 / 1.3478840$

[44] Q. Qiao, D. Xu, Y. W. Li, J. Z. Zhang, Z. G. Hu, and J. H. Chu, "Detection of Resistive Switching behavior based on the $\mathrm{Al}_{2} \mathrm{O}_{3} / \mathrm{ZnO} / \mathrm{Al}_{2} \mathrm{O}_{3}$ Structure with Alumina Buffers," Thin Solid Films, vol. 623, pp. 8-13, Feb 2017. doi: 10.1016/j.tsf.2016.12.053

[45] D. Kumar, R. Aluguri, U. Chand, and T. Y. Tseng, "Role of $\mathrm{Al}_{2} \mathrm{O}_{3}$ Thin Layer on Improving the Resistive Switching Properties of $\mathrm{Ta}_{5} \mathrm{Si}_{3}$-Based Conductive Bridge Random Accesses Memory Device," Jpn. J. Appl. Phys., vol. 57, no. 4, p. 04FE16, Apr 2018. doi: 10.7567/jjap.57.04fe16

[46] R. Waser, R. Dittmann, G. Staikov, and K. Szot, "Redox-Based Resistive Switching Memories - Nanoionic Mechanisms, Prospects, and Challenges," Adv. Mater., vol. 21, no. 25-26, pp. 2632-2663, Jul 132009. doi: 10.1002/adma.200900375

[47] M.-C. Chen, T.-C. Chang, C.-T. Tsai, S.-Y. Huang, S.-C. Chen, C.-W. Hu, S. M. Sze, and M.-J. Tsai, "Influence of Electrode Material on The Resistive Memory Switching Property of Indium Gallium Zinc Oxide Thin Films," Appl. Phys. Lett., vol. 96, no. 26, p. 262110, 2010. doi: $10.1063 / 1.3456379$

[48] T. Thuy Trinh, V. Duy Nguyen, H. Hanh Nguyen, J. Raja, J. Jang, K. Jang, K. Baek, V. Ai Dao, and J. Yi, "Operation Mechanism of Schottky Barrier Nonvolatile Memory with High Conductivity InGaZnO Active Layer," Appl. Phys. Lett., vol. 100, no. 14, p. 143502, 2012. doi: 10.1063/1.3699221

[49] E. W. Lim and R. Ismail, "Conduction Mechanism of Valence Change Resistive Switching Memory: A Survey," Electronics, vol. 4, no. 3, pp. 586-613, Sep 2015. doi: 10.3390/electronics4030586 